Beatrix Borchard / Monika Schwarz-Danuser (Hrsg.)

Fanny Hensel geb. Mendelssohn Bartholdy 


\author{
Beatrix Borchard / \\ Monika Schwarz-Danuser (Hrsg.)
}

\title{
Fanny Hensel \\ geb. Mendelssohn Bartholdy
}

Komponieren zwischen Geselligkeitsideal und romantischer Musikästhetik

Verlag J. B. Metzler

Stuttgart - Weimar 
Die Deutsche Bibliothek - CIP-Einheitsaufnahme

Fanny Hensel geb. Mendelssohn Bartholdy : Komponieren zwischen Geselligkeitsideal und romantischer Musikästhetik /

Beatrix Borchard/Monika Schwarz-Danuser. Stuttgart ; Weimar : Metzler, 1999

(M-\&-P-Schriftenreihe für Wissenschaft und Forschung)

ISBN 978-3-476-45204-7

ISBN 978-3-476-45204-7

ISBN 978-3-476-04298-9 (eBook)

DOI 10.1007/978-3-476-04298-9

Dieses Werk ist einschließlich aller seiner Teile geschützt. Jede Verwertung außerhalb der engen Grenzen des Urheberrechtsgesetzes ist ohne Zustimmung des Verlages unzulässig und strafbar. Das gilt insbesondere für die Vervielfältigungen, Übersetzung, Mikroverfilmungen und Einspeicherung in elektronischen Systemen.

M \& P Schriftenreihe für Wissenschaft und Forschung

(C) 1999 Springer-Verlag GmbH Deutschland

Ursprünglich erschienen bei J.B. Metzlersche Verlagsbuchhandlung und Carl Ernst Poeschel Verlag GmbH in Stuttgart 1999 


\section{An Fanny}

Vom Himmel fiel ein heller Tag hernieder,

Mit seinem Blumenschmucke angethan

War schon der grüne Wiesenplan,

Und die Nachtigall sang ihre Sommerlieder,

Da trat ein holdes Kind in diese Welt,

Und lächelte und freute sich im bunten Frühlingszelt.

Die zarten Geister, die in den Kristallen

Der Flüsse wohnen, die in Luft,

In Sommerwind, in Blüthenduft,

In kühlen Lauben, grünen Blätter fallen,

Sie kamen alle spielend hergezogen,

Und sangen süßen Gruß: "wir sind dem schönen Kind gewogen."

Sieh, wie sich dir entgegen neigt gelinde

Der hellen Lilien weiße Pracht,

Wie dir entgegen Rose lacht,

Das Veilchen blau, das liebliche Gesinde

Das Frühling mit sich bringt zu seinen Scherzen,

Die Aeuglein süß, die Lippen roth, die brennend goldnen Kerzen.

Sie alle dienen dir, die bunten Strahlen,

Die still und zärtlich nach dir blicken,

Und dich mit lieben Duft erquicken,

Sie alle wollen nur dein Bildniß mahlen;

Doch Anmuth und den Reiz die dich umschweben,

Ermangeln sie der Mahlerkunst, drum stirbt ihr flüchtig Leben.

Wir schenken dir der Nachtigallen Klänge,

Daß du mit zartem Lautenspiele,

Kannst singen deiner Brust Gefühle,

Und gern vernimmt der Dichtenden Gesänge,

Drum horche deines Dichters Weisen,

Wird er des Liebesgotts Gewalt und deine Schönheit preisen.

Ludwig Tieck

(gewidmet Fanny Hensel) 


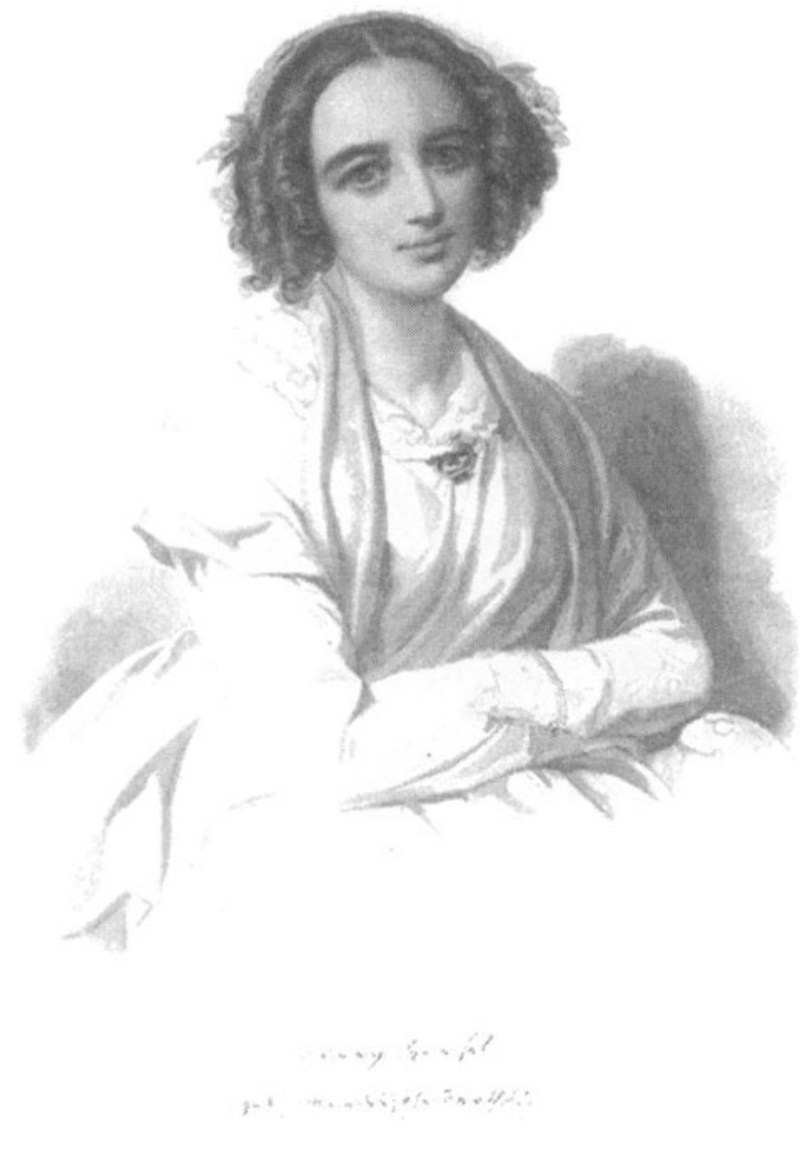




\section{Inhaltsverzeichnis}

\section{Vorwort}

Beatrix Borchard

Ein Rufen nur aus Träumen?.....................................................................

Monika Schwarz-Danuser

Desiderata der Forschung

XIX

\section{Der Salon als sozialer und kultureller Ort}

Barbara Hahn

Häuser für die Musik

Akkulturation in Ton und Text um 1800 .....................................................

Beatrix Borchard

Opferaltäre der Musik

\section{Experimentelles Komponieren}

Peter Rummenhöller

Lied ohne Worte op. 8,2

Cornelia Bartsch

Das Lied ohne Worte op. 6,1 als offener Brief.

Christian Thorau

"Das spielende Bild des Jahres“

Fanny Hensels Klavierzyklus Das Jahr 
Annette Nubbemeyer

Die Klaviersonaten Fanny Hensels

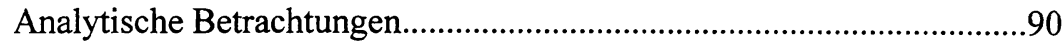

Renate Hellwig-Unruh

Zur Entstehung von Fanny Hensels

Streichquartett in Es-Dur (1829/34).

Rainer Cadenbach

"Die weichliche Schreibart", "Beethovens letzte Jahre"

und "ein gewisses Lebensprinzip"

Perspektiven auf Fanny Hensels spätes Streichquartett (1834).

Gesine Schröder

Schreiben für Orchester.

\section{Tradieren und Edieren}

Barbara Gabler

Tradieren und Edieren aus Verlagssicht.

Annette Maurer

Überlegungen zur Edition der Werke Fanny Hensels

Diether de la Motte

... als später Gehilfe der zu eiligen Komponistin

\section{Bachrezeption}

Janina Klassen

Fugenfinger und reine Kunst

Hintergründe der Bach-Rezeption Fanny Hensels.

Susanne Fontaine

Bach, das Gegenbild 
Hans-Joachim Hinrichsen

Choralidiom und Kunstreligion

Fanny Hensels Bach. .216

Ellinore Fladt

Das problematische Vorbild

Zur Rezeption des "vokalen Bach" in der Kantate Hiob.

\section{Zur Biographie}

Françoise Tillard

Felix und Fanny Mendelssohn Bartholdy -

Verkörperung bürgerlicher Perfektion.

Marian Wilson Kimber

Zur frühen Wirkungsgeschichte Fanny Hensels.

\section{Zwischen Judentum und Christentum}

Julius H. Schoeps

Christliches Bekenntnis oder modernes Marranentum?

Der Übergang vom Judentum zum Christentum:

Das Beispiel Abraham und Felix Mendelssohn Bartholdy. .265

Christian Knopf

Synagoge und Ecclesia.

Wolfgang Dinglinger

"Die Glaubensform der meisten gesitteten Menschen"

Aspekte der christlichen Erziehung der Geschwister Mendelssohn......288

Eva-Maria Thimme

"Gedanken gehn und Lieder fort bis ins Himmelreich"

Fanny Hensel, die jüdische und die christliche Tradition. 
Monika Schwarz-Danuser

"Weihrauchdampf" und "Kopfhängerei"

Fanny Hensels belastetes Verhältnis zur katholischen Kirchenmusik..312

Kurzbiographien der Autorinnen und Autoren..............................329

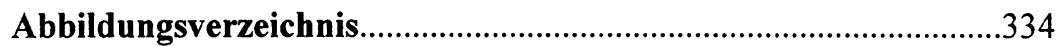

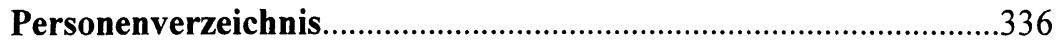




\section{Ein Rufen nur aus Träumen?}

Wer sich heute mit den Werken von Fanny Hensel beschäftigt, ist sofort mit Grundsatzfragen konfrontiert. Es beginnt schon mit dem Namen. ${ }^{1}$ Wie sie nennen: Fanny Mendelssohn? Fanny Mendelssohn Bartholdy? Fanny Hensel? Fanny Hensel-Mendelssohn? Fanny Hensel-Mendelssohn-Bartholdy? Oder auch nur Fanny? Für die ersten drei Versionen gibt es Argumente, der erste ist ihr Geburtsname, der zweite ihr Taufname, zu dem auch noch der neue Vorname, nämlich Cäcilie hinzukam. Den dritten schließlich führte sie ab ihrer Eheschließung, unter ihm publizierte sie auch. Keine Argumente gibt es für den Doppelnamen. Gleich in welcher Gestalt, ist er eine Konstruktion aus der Sicht von heute und verknüpft im Verkaufsinteresse von Schallplattenfirmen und Buchverlagen den unbekannten Komponistennamen Hensel mit einem berühmten, dem Namen des Bruders, Felix Mendelssohn Bartholdy. Die Anrede Fanny schließlich schien uns Ausdruck unangemessener Familiarität, die leider im Umgang mit den Namen von Frauen weitverbreitet ist. Also haben wir uns auf den Namen Fanny Hensel geb. Mendelssohn Bartholdy, kurz Fanny Hensel ${ }^{2}$ geeinigt und den unterschiedlichen Namensgebrauch unserer Autoren und Autorinnen in diesem Sinne vereinheitlicht.

Während die Namensfrage qua Vereinbarung relativ leicht zu lösen ist, stellt sich die Werkfrage sehr viel komplexer dar. Denn was ist das Werk von Fanny Hensel? Alles, was sie jemals komponiert hat? Nur die wenigen

1 vgl. zur Namensproblematik besonders bei jüdischen Autorinnen Barbara Hahn, Unter falschem Namen, Frankfurt/Main 1991

2 Der amerikanischen Tradition, nur den Nachnamen als Autorennamen zu nennen, wollten wir uns nicht anschließen, weil der Nachname Hensel für ihren Mann Wilhelm Hensel steht. 
Werke, die sie selber noch veröffentlichen konnte, also op.1-7, einschließlich der Stücke, die sie bzw. ihr Bruder nach ihrem Tode noch zum Druck vorbereitet hat, also einschließlich der Opusnummern 8-11? Der größte Teil dessen, was Fanny Hensel komponiert hat, ist uns nur in mit vielen Korrekturen übersäten Entwurfsfassungen überliefert, und selbst zu umfangreichen Stücken wie ihren drei Kantaten, die sie nachweislich zur Aufführung gebracht hat, fehlen Reinfassungen. Einer Handvoll autorisierter Opera in den Gattungen klavierbegleitetes Sololied, Klavierlied, Chorlied, Klaviertrio stehen ca. 450 weitere Stücke auch anderer Gattungen gegenüber, von denen wir nicht wissen, ob Fanny Hensel mit ihrer Veröffentlichung einverstanden gewesen wäre, und wenn ja, in welcher Werkgestalt.

Die Frage nach Fanny Hensels Werk löst eine Fülle von Überlegungen aus, die den Rahmen üblicher Editionsproblematik sprengen und die für die Wissenschaft eine interessante Herausforderung in sich bergen. Denn jede posthume Veröffentlichung ist mit der Konstruktion einer Werkgestalt verbunden, die durch die Komponistin selber nicht autorisiert ist. Das zwingt uns z.B., über unseren Werkbegriff, über Autorschaft, die Bedeutung von Entstehungs- und Aufführungsbedingungen für ihre Werke, der Adressierung ihrer Musik etc. neu nachzudenken.

Bisher ist man mit der Editionsfrage eher pragmatisch umgegangen. Der Furore-Verlag Kassel, in dem der größte Teil des Nachlasses in erster Linie von ausübenden Musikerinnen und Musikern publiziert worden ist, wollte spielbare Fassungen an die Öffentlichkeit bringen. Entsprechendes gilt für den Druck von einzelnen Liedern und mehrstimmigen Vokalwerken, der Vertonung der ersten Szene aus dem zweiten Teil des Faust und für die $M u$ sik zum Feste der heiligen Cäcilia, die Suzanne Summerville (Arts Venture) herausgebracht hat. Je nach Zustand der Autographe war bei diesen Ausgaben der Ermessensspielraum für die jeweiligen Editoren und Editorinnen unterschiedlich groß: Der Herausgabe von Liedkompositionen mit nur geringen Korrekturen steht der Druck umfangreicher Werke wie z.B. des so- 
genannten Oratoriums, der Cantate. Nach Aufhören der Cholera in Berlin 1831 gegenüber. Fanny Hensel hat die Cantate zwar aufgeführt, aber das Autograph weist so viele Korrekturen auf, daß oft nicht eindeutig zu rekonstruieren ist, für welche Version nicht nur einzelner Abschnitte sondern ganzer Sätze sie sich selber entschieden hat. Inzwischen sind Forderungen nach revidierten und kritisch kommentierten Ausgaben laut geworden, für einen Teil der Lieder (Annette Maurer) und auch der Klavierstücke (Annegret Huber) liegen sie auch bereits vor.

Die Beiträge des vorliegenden Buches zeigen, wie groß aufgrund der skizzierten Ausgangssituation der Diskussionsbedarf vor allem über aufführungspraktische Probleme, den Werkcharakter, Fragen der Analyse und musikhistorischen Einordnung der in den letzten Jahren veröffentlichten Werke von Fanny Hensel ist. Sie dokumentieren das erste internationale Symposion, das dem Werk Fanny Hensels gewidmet war und das von Beatrix Borchard (Hochschule der Künste Berlin) und Monika Schwarz-Danuser (Erzbischöfliche Kirchenmusikschule Berlin) vom 28.-30. November 1997 an der Hochschule der Künste Berlin veranstaltet worden ist. Ziel des Symposions war eine erste Bestandsaufnahme der Fanny-Hensel-Forschung ${ }^{3}$ und die gemeinsame Entwicklung weiterführender Fragestellungen. So saßen denn alle an einem Tisch - die Pionierinnen der Fanny-HenselForschung und junge Forscherinnen, Interpreten und Hörer, Bewunderinnen und Skeptiker und die etablierte Musikwissenschaft.

In der insgesamt noch sehr jungen Fanny Hensel Forschung hat sich in den letzten Jahren ein Generationswechsel vollzogen. Waren es zunächst ausübende Musikerinnen, die dafür gesorgt haben, daß Werke aufgeführt, gedruckt und eingespielt wurden, gibt es nun eine ganze Reihe von Nachwuchswissenschaftlerinnen, die mit ersten Magisterarbeiten und Promotio-

3 Zum Symposion erschien eine erste von Martina Helmig herausgegebene Aufsatzsammlung unter dem Titel Fanny Hensel geb. Mendelssohn Bartholdy, Das Werk, München 1997. 
nen analytische Arbeiten vorgelegt haben. Der Dialog zwischen diesen beiden Generationen, der nicht zuletzt auch ein Dialog zwischen Praxis und Wissenschaft ist, war längst überfällig und sehr fruchtbar.

Die thematischen Schwerpunkte ergaben sich aus dem bisherigen Forschungsstand. Gegenstand ausführlicher Darlegungen war "Der Salon als geistiger und sozialer Ort", "Experimentelles Komponieren“, "Biographik zwischen Konstruktion und Rekonstruktion". Sie wurden teilweise für dieses Buch noch einmal erweitert und aufgrund der Diskussion revidiert. Weitere zentrale Themen wie Editionsfragen, Bachrezeption, Fanny Hensel zwischen Judentum und Christentum wurden aus verschiedenen Perspektiven und Arbeitszusammenhängen heraus auf der Basis kurzer Statements dargestellt und mit dem zahlreichen und sehr informierten Publikum diskutiert. Wir haben versucht, den Charakter dieser Gesprächsrunden so weit wie möglich zu erhalten, und da es sich um einen Kongreßbericht handelt, haben wir so wenig wie möglich in die Texte der einzelnen Autoren und Autorinnen eingegriffen.

Ausgangspunkt des Symposions und auch der vorliegenden Publikation war der Versuch, den "Mythos vom Salon" (Barbara Hahn) zu hinterfragen, den Salon als sozialen und kulturellen Ort im Kontext des jüdischen Akkulturationsprozesses um 1800 neu zu beschreiben und nach der Rolle der Musik in diesem Prozeß zu fragen. Es ist bekannt, daß bereits eine Generation später das Mendelssohnsche Haus für das Berliner Musikleben zentral wurde, aber gemeinhin setzt man die Adresse Leipziger Straße Drei mit dem berühmten Gartensaal als halböffentlichem Aufführungsort gleich. Tatsächlich jedoch umfaßte die Leipziger Straße mehrere Aufführungsräume ganz unterschiedlichen Charakters, Räume, mit denen auch unterschiedliche ästhetische Konzepte verbunden waren (Beatrix Borchard).

Mit dem provokativen Titel "Experimentelles Komponieren", ein Begriff, den wir eher mit der Musikentwicklung des 20. als mit der des 19. Jahrhunderts verbinden, wollten wir die Bedingungen, die die Arbeit Fanny 
Hensels geprägt haben, und ihre Kompositionshaltung charakterisieren. Da in Kürze zwei Dissertationen über die Lieder Fanny Hensels (Annette Maurer und Gisela Müller) vorliegen werden, konzentrierten wir uns auf Werke anderer Gattungen, ihre Lieder ohne Worte (Peter Rummenhöller und Cornelia Bartsch), den Klavierzyklus Das Jahr (Christian Thorau), die Klaviersonaten (Annette Nubbemeyer), ihr Streichquartett (Renate Hellwig-Unruh, Rainer Cadenbach), schließlich auf ihre Orchesterkompositionen (Gesine Schroeder).

In den einzelnen Beiträgen zeichnen sich deutlich verschiedene Haltungen $\mathrm{ab}$, mit denen die einzelnen Referenten und Referentinnen an die Musikanalyse herangegangen sind. So bewertet der eine Fanny Hensels Umgang mit thematischem Material, ihr "modulierendes Infragestellen des musikalischen Gedächtnisses" (Diether de la Motte) als Novität, der andere hingegen beklagt die mangelnde Prägnanz der musikalischen Gedanken (Rainer Cadenbach). Diese unterschiedlichen Einschätzungen spiegeln die Schwierigkeiten, auf die der Versuch einer Einordnung des Oeuvres von Fanny Hensel in die deutsche Musikgeschichte der ersten Hälfte des 19. Jahrhunderts allenthalben stößt. Wer über Beethoven spricht und schreibt, bewegt sich auf einem gesicherten Terrain, schreibt sich ein in die tradierte Musikgeschichtsschreibung, wer hingegen über Fanny Hensel nachdenkt, muß seine Kriterien überprüfen und zur Diskussion stellen. Wie schwer es ist, musikalische Sachverhalte jenseits von Bewertungen zu beschreiben, wurde in den Diskussionen und Beiträgen immer wieder deutlich, und so stellte sich auch beim Thema "Bachrezeption" die Frage der Kriterien, mit denen wir uns der Musik Fanny Hensels nähern: Nachahmung oder eigenständige Auseinandersetzung - Experiment im Sinne eines Beitrages zur "Problemgeschichte des Komponierens" oder vollgültiges Werk (Janina Klassen, Susanne Fontaine, Hans-Joachim Hinrichsen, Ellinore Fladt).

Für die Thematisierung der bisherigen Editionspraxis (Barbara Gabler, Annette Maurer) haben wir bewußt die aktive Verbform "Edieren" gewählt, 
ging es doch nicht nur um die Endprodukte in Gestalt gedruckter Werke, sondern um die Thematisierung des Editionsprozesses selber. Einen der Pole im Umgang mit der musikalischen Hinterlassenschaft Fanny Hensels markiert Diether de la Motte, Fanny Hensel Enthusiast der ersten Stunde, der "...als später Gehilfe der zu eiligen Komponistin" z.B. Quintparallelen korrigiert und damit in den Notentext selber eingreift, einen anderen das Fernziel einer historisch-kritischen Gesamtausgabe, in der die verschiedenen Notentextschichten dokumentiert werden könnten.

Eng verbunden mit dem Thema "Edieren" ist das Thema "Tradieren", sind doch für die Wirkungsgeschichte von Fanny Hensels Werken, abgesehen von dem Niederschlag, den ihre Musik im musikalischen Denken des Bruders gefunden hat, andere Tradierungsformen als die Verbreitung über den Druck kennzeichnend. Um sie zu erfassen, muß man sich noch genauer als bisher geschehen mit zeitgenössischen Kommunikationsformen auseinandersetzen. So ist ein Teil der Stücke über Abschriften, Briefe, Stammbucheintragungen, oder auch als anonymer Abdruck in Almanachen verbreitet worden.

Werk und Biographie sind nicht voneinander zu trennen, und so wurden, wenn auch im Zentrum des Symposions die kompositorische Arbeit von Fanny Hensel stand, biographische Fragen immer wieder thematisiert, so z.B. von der ersten Fanny Hensel Biographin, der französischen Pianistin und Musikwissenschaftlerin Françoise Tillard (Paris), die in ihrem Beitrag den enormen Anpassungsdruck, unter dem die beiden Geschwister standen nachzeichnete, und durch Marian Wilson Kimber, die überraschendes Material zur frühen Rezeptionsgeschichte von Fanny Hensel in England, in den USA und in Frankreich vorstellte.

Mit dem Thema "Zwischen Judentum und Christentum" schließt sich gewissermaßen der Kreis - ein Thema, das kaum eindeutige Antworten, aber eine Fülle interessanter Fragen aufwirft, so z.B. zur Funktion und Folge der Konversion vom Judentum zum Protestantismus (Julius H. Schoeps), 
zum Verhältnis von Christentum und Judentum (Christian Knopf), zum Religionsverständnis und Erziehungskonzept Abraham Mendelssohns (Wolfgang Dinglinger), zu Fanny Hensels Verhältnis zum Katholizismus (Monika Schwarz-Danuser) und zur Rolle der Frauen im Judentum (Eva-Maria Thimme).

Aus der Fülle der hier abgedruckten Beiträge entsteht wie in einem Kaleidoskop ein Fanny Hensel Bild, das nicht festgeschrieben ist, sondern, je nachdem welche Frage man akzentuiert, sich immer wieder neu zusammensetzt.

Fanny-Hensel-Briefmarke - Fanny-Hensel-Denkmal in Hamburg, FannyHensel-Weg in Berlin - Fanny-Hensel-Grundschulen - man mag solche Taufaktionen für oberflächlich erachten, aber sie dienen doch dazu, einen Namen in das öffentliche Bewußtsein zu bringen. Ist ein Name etabliert, dann kann man nicht zuletzt auch leichter Geld beantragen für wissenschaftliche Forschung, für Aufführungen etc. Die große Resonanz auf unser Symposion sowohl von Seiten des Publikums als auch von den Medien hat gezeigt, daß inzwischen ein breites öffentliches Interesse an der Musik Fanny Hensels wach geworden ist. Dennoch stehen wir erst am Anfang der Fanny-Hensel-Forschung, noch sind nicht alle biographischen Quellenmateralien erschlossen (vor allem fehlt noch eine Edition der Tagebücher), nicht alle Kompositionen ediert bzw. die ersten Ausgaben revidiert.

Das Symposion war Teil eines von der Stiftung Deutsche Klassenlotterie finanzierten Gesamtprojekts anläßlich des 150. Todestages, das von der Musikjournalistin Martina Helmig und der Dirigentin Barbara Kaiser in $\mathrm{Zu}-$ sammenarbeit mit Musikfrauen e.V. Berlin initiiert worden war. Es wurde ermöglicht durch die Senatsverwaltung für Arbeit, Berufliche Bildung und Frauen, der ebenso unserer besonderer Dank gilt, wie der Hochschule der Künste, die die Werbekosten übernommen hat, und ihrem Zentrum für Interdisziplinäre Frauenforschung und Gender Studies. Die Druckkosten für den vorliegenden Symposionsbericht stellte dankenswerterweise ebenfalls 
die Stiftung Deutsche Klassenlotterie zur Verfügung. Vor allem aber danken wir allen Kollegen und Kolleginnen, die überwiegend unentgeltlich an dem Symposion mitgewirkt haben. Ebenso gilt unser Dank Hartmut Fladt, Sabine Vogt-Schneider und Bettina Brand für die Unterstützung unserer Redaktionsarbeit.

Berlin, den 28. September 1998

Beatrix Borchard 


\section{Desiderata der Forschung}

Im Lichte der Tatsache, daß in den vergangenen Jahren und Jahrzehnten die musikwissenschaftlichen Methodologien um "Gender Studies" substantiell erweitert worden sind, fallen einige Desiderata der FannyHensel-Forschung mit aktuellen Perspektiven der "Gender Studies" zusammen. Auch wenn die Kategorie der menschlichen und kompositorischen Individualität auf diese Komponistin und Musikerin bezogen werden muß, so bietet sie doch aufgrund der Geschwisterkonstellation mit Felix Mendelssohn Bartholdy für vergleichende Geschlechterstudien ein besonders interessantes und fruchtbares Fallbeispiel, mutatis mutandis in einigen Aspekten der Geschwister-, wenn nicht gar Zwillingsforschung in Biologie bzw. Medizin vergleichbar. Gerade weil eine auf die Werkkategorie gestützte Musikhistoriographie mittlerweile keineswegs mehr das einzig mögliche oder auch nur das dominierende Paradigma darstellt, eröffnen sich aus der engen Verbindung zwischen Fanny Hensel und ihrem Bruder Felix Mendelssohn Bartholdy Perspektiven auf eine dialogische Musikgeschichte, worunter nichts anderes zu verstehen wäre als eine Rekonstruktion der Werke aus der Situation eines engen persönlichen, kritischen Kontaktes im Mendelssohnschen Familienkreis. ${ }^{1}$

Eine über dieses Symposion hinaus wichtige Aufgabe liegt darin, analytisch angemessene Kriterien für ein Verständnis der Kompositionen Fanny Hensels zu entwickeln, Kriterien, die dem besonderen Charakter des sozialen Bezugsnetzes ihrer Kunst spezifisch Rechnung tragen. Es wäre von einer Bildungs- und Geselligkeitskultur auszugehen, wie sie sich in den von Fanny Hensel gepflegten Gattungen Lied, Klavier- und Kammermusik ${ }^{2}$ ausdrückt und die zweifellos mit ihrer Rolle als Frau zu-

1 Vgl. dazu die Beiträge von Cornelia Bartsch und Rainer Cadenbach in dieser Publikation.

2 Zu dieser Art Geselligkeitskultur gehören neben den genannten Gattungen, die als opusfähig gelten, auch die musikalischen Beiträge zu beliebten Geselligkeitsfor- 
sammenhängt. Der Kunstcharakter ihrer Kompositionen ist im allgemeinen nicht gegen, sondern im Verbund mit einem Geselligkeitsideal subtilerer Art entwickelt worden. Auch liegt es nahe, die von ihr für das Liedschaffen gewählten Texte interdisziplinär im gemeinsamen Studium mit Literaturwissenschaftlern zu bewerten, um dem ganz eigenständigen Charakter dieser Kunst bürgerlicher Verinnerlichung nahezukommen. Dabei wird es aber nicht sinnvoll sein, das reiche Liedschaffen über einen einzigen Kamm zu scheren, Ziel müßte es vielmehr sein, innerhalb dieses Oeuvres verschiedene Richtungen des Komponierens und Musizierens zu beschreiben - bezogen sowohl auf eine Psychosozialgeschichte der bürgerlichen Hausmusik und der halböffentlichen Sonntagsmusiken, als auch auf den Wunsch, eigene Werke publizieren zu dürfen.

Was die musikalische Analyse selbst anbetrifft, so hat sie in Zukunft noch vermehrt dem Umstand Rechnung zu tragen, daß der größere Teil von Fanny Hensels Schaffen, wie Beatrix Borchard oben bereits hervorhob, einen Zustand der Werkdurchbildung widerspiegelt, der nicht von der Autorin als für den Druck genehmigt gelten kann. Es ist ja zur Genüge bekannt, in welchem Maße Komponisten ihre Werke im Zuge der Drucklegung noch veränderten und glätteten. Die Analyse hat demzufolge immer eines hypothetischen Charakters der Textgestalt eingedenk zu bleiben. Umgekehrt kann sie aber mit um so größerem Recht die Texte dieser Werke in ihrem momentan gegebenen, durch die private oder halböffentliche Aufführungen sanktionierten Wirkungszusammenhang untersuchen und ihre Funktion in dieser Rolle aufdecken, weil die Musik aufgrund ihrer unveröffentlichten Textgestalt ihren "Sitz im Leben" unmittelbarer als in gedruckter Form offenbart.

In diesem Kontext gewinnen auch Fanny Hensels Briefe und Tagebücher besondere Aussagekraft, zeigen sie nämlich eine Autorin, die sich

men des frühen 19. Jahrhunderts wie Lebende Bilder, Charaden, Festspiele und ähnliches. Diese werden in Fanny Hensels bisher vorliegenden Tagebuchaufzeichnungen und Briefen ausführlich beschrieben. 
spontaner äußert als ihr Bruder Felix, eine Schriftstellerin, deren Texte als Dokumente einer inneren und äußeren Lebensgeschichte sowie als Quellen für ihre explizite musikalische Poetik noch keineswegs ausreichend erschlossen sind.

Da Bildung in dieser Epoche insbesondere als ein Prozeß innerer Reifung verstanden wurde, umfassen die Komponenten dieses musikalischen Programms neben den "geselligen", auf eine dialogische, fröhliche Unterhaltung im geistreichen Austausch zielenden Faktoren stets auch ethische, denn die moralische Bildung soll durch die kulturelle und künstlerische Praxis mitbefördert werden. Die Ästhetik erweist sich demnach, in dieser Hinsicht, noch keineswegs als eine "autonome" Sphäre, gerade ihre dominierende Rolle legitimiert sich mit Bezug auf eine moralische, soziale Qualität.

Ebenso ist die Bildungsreise nach Italien, die Fanny Hensel mit ihrer Familie 1839-1840 unternahm, im Kontext der verbreiteten Italienreisen seit dem 18. Jahrhundert noch weiter zu erforschen. Gerade hier bieten sich Möglichkeiten eines interessanten Vergleichs zwischen den Geschwistern Mendelssohn Bartholdy. Die literarische Prägung dessen, was als Reiseerlebnis sich einstellt, ist vor dem Hintergrund der geistigen Vaterfigur Goethe zu bewerten - Eigenes und Fremdes durchdringen sich hier in einer schwer entwirrbaren Weise -, aber auch die kompositorischen Reflexe der Reisen beider Geschwister wären vergleichend zu bedenken. Während bei Felix Mendelssohn Bartholdy aus den Bildungsreisen nach Paris und Italien eine verstärkte Besinnung auf seine eigene Existenz als deutscher Komponist resultierte, ${ }^{3}$ wird bei Fanny Hensel neben diesen auch bei ihr vorhandenen Komponenten ein verstärktes

3 vgl. Norbert Miller, Felix Mendelssohn Bartholdys italienische Reise, in: Das Problem Mendelssohn, hg. v. Carl Dahlhaus (= Studien zur Musikgeschichte des 19. Jahrhunderts, Bd. 41), Regensburg 1974, S. 23-35, sowie Rudolf Stephan, Über einige Ansichten, Felix Mendelssohn Bartholdy betreffend, in: Felix Mendelssohn Bartholdy. Kongreß-Bericht Berlin 1994, hg. v. Christian Martin Schmidt, Wiesbaden 1997, S. 12-17 
Sich-Öffnen gegenüber dem Fremden sichtbar, dessen Bedeutung für ihr Selbstverständnis als Komponistin noch näher zu ergründen wäre.

Insgesamt, so scheint es, könnte die Fanny-Hensel-Forschung noch profitieren von einem engeren Austausch mit der aktuellen GoetheForschung, wie sie derzeit nicht zuletzt durch Klassiker-Editionen im Hinblick auch auf die kunsttheoretischen und ästhetischen Schriften Goethes einen neuen Schub erfahren hat. Diese überragende Figur war für die Geschwister Mendelssohn sowohl direkt wie indirekt - über Karl Friedrich Zelter, dessen Profil in der Korrespondenz mit Goethe klar hervortritt - von entscheidender Bedeutung. Dabei wäre noch näher zu untersuchen, wie die musikalische Unterweisung der Geschwister Fanny und Felix im einzelnen sich gestaltete, ist doch keineswegs davon auszugehen, daß diese völlig parallel verlaufen ist. ${ }^{4}$

Welchen Stellenwert nimmt Fanny Hensel, so wäre weiter zu fragen, innerhalb der Rezeptionsgeschichte von Goethes Faust durch die Komposition ihrer Faust-Szene ein? Welche Anregungen musikalischer Art verarbeitet sie darin? Hat sie umgekehrt in diesem Sinne allenfalls sogar auf Charles Gounod eingewirkt, dessen Oper Faust nach Berlioz' fantastisch-satanischer Adaptation die lyrische Dimension der Liebesgeschichte entfaltet? Auch hier wird die Fanny-Hensel-Forschung mit Gewinn an die jüngere Diskussion der Literaturwissenschaft anknüpfen können, ja sie wird, gerade was Gounods Äußerungen über Fanny Hensel anbetrifft, ${ }^{5}$ zu fragen haben, ob hier nicht ein Fallbeispiel jener

4 Verwiesen sei auf den Aufsatz von Gesine Schröder in diesem Band.

5 Charles Gounod war im Jahre 1840, als Fanny Hensel sich mit ihrer Familie in Rom aufhielt, Rompreis-Stipendiat in der Villa Medici und häufiger Gast der Familie Hensel. Hier lernte er Bach, Beethoven, die neuesten Kompositionen von Felix und Fannny kennen sowie vermutlich auch Goethes Faust. Wie sehr ihn diese künstlerischen Impulse aufgewühlt haben, hat Fanny Hensel in ihrem Tagebuch treffend beschrieben, desgleichen die ungewöhnliche Intensität von Gounods Besuch in Berlin einige Jahre später (vgl. Françoise Tillard, Die verkannte Schwester. Die späte Entdeckung der Komponistin Fanny Mendelssohn Bartholdy, München 1996, S. 468 [frz. Originalausgabe Paris 1992]). Dagegen wirken die Bemerkungen, die Charles Gounod selbst in seinen Mémoires d'un artiste über Fanny Hensel 
"Anxiety of Influence "6 vorliegt, mit welchem Begriff der amerikanische Literaturwissenschaftler Harold Bloom das Verschweigen oder Herunterspielen gerade der dominierenden künstlerischen Einflüsse zu einer Theorie literarischer Rezeption geformt hat. Es ist keineswegs auszuschließen, daß Einflüsse, die von Frauen ausgingen, von männlichen Künstlern besonders hartnäckig verschwiegen wurden - es sei denn auf der Ebene der Funktion der Muse und Inspirationsgeberin -, weil andernfalls die männliche Form künstlerischer Produktivität ihren Ausschließlichkeitsanspruch hätte preisgeben müssen. Hier eröffnet sich noch ein weites Feld der Forschung, und zwar um so mehr, als offenbar in Frankreich Fanny Hensels Reputation insgesamt bedeutender war, als bisher angenommen werden konnte. ${ }^{7}$

formulierte, im Hinblick auf den intellektuellen Austausch und die von ihr empfangenen künstlerischen Anregungen merkwürdig distanziert (vgl. Françoise Tillard, ebd., S. 432).

Immerhin ist Fanny Hensel im Grand Dictionaire universel du XLX Siècle von Pierre Larousse bereits 1873, also noch vor Erscheinen von Sebastian Hensels Buch Die Familie Mendelssohn 1729-1847. Nach Briefen und Tagebüchern mit einem eigenen Artikel vertreten. Dort heißt es: Hensel (Fanny Mendelssohn, dame), pianiste et compositeur allemand, femme du précédant et soeur de Mendelssohn, née à Hambourg vers 1808, morte à Berlin en 1847. Pianiste du premier ordre, musicienne exquise et profondément instruite dans son art, elle composa, toute jeune encore, des romances sans paroles pour le piano, et des lieders que son illustre frère eut pû signer sans compromettre son nom; car, dans ses compositions musicales, à la fermeté de la pensée et à la netteté du dessin se joignent une élégance et un charme tout féminins. Cependant, malgré la réunion de toutes ses qualités, qui lui eussent assuré la gloire, cette délicate créature qu'effrayent les manifestations bruyantes de la popularité, ne voulut jamais produire son talent hors du foyer de la famille et d'un petit cercle d'amis. Ce n'est qu'à force d'instances et de prières que son illustre frère parvint à vaincre cette farouche timidité, et obtint son consentement à la publication de ses æuvres; mais, le 14 mai 1847, juste le lendemain du jour où ses compositions étaient mises en vente chez l'éditeur, Fanny Hensel était enlevée par une morte précoce. Cette perte frappa tellement Mendelssohn que, six mois après, il suivit dans la tombe cette soeur bien aimée. Les productions de Madame Hensel qui ont été publiées sont: dix-huit lieders pour voix, avec accompagnement de piano, quatorze lieders sans paroles, et douze mélodies pour piano [9. Bd., Paris 1873, S. 194]. Bei dem Buch von E. Sergy [Noëmi Kö- 
Wie läßt sich ferner das musikalisch-schöpferische Profil Fanny Hensels über die im vorliegenden Band vereinigten neueren Ergebnisse hinaus noch weiter historisch perspektivieren? Während Beethoven als Leitfigur eines künstlerisch begründeten Humanismus fungierte, hatte die Bach-Rezeption - vermittelt durch Zelter und die Familientradition im Hause Mendelssohn Bartholdy - eine künstlerisch-religiöse Doppelfunktion. Indem Bachs Oeuvre verinnerlicht wurde, leistete Fanny Hensel zugleich einen Beitrag zur jüdischen Akkulturation an den in Preußen herrschenden Protestantismus. Wenn hier die Quellen noch weiter erschlossen und interpretiert werden, eröffnen sich fruchtbare Perspektiven auf eine Biographik, welche musikalische, literarische und religiöse Quellen in ihrer wechselseitigen Einflußnahme zu deuten sucht. Auch das Verhältnis zu Hegels Religionsphilosophie und zur Gefühlsreligion seines Antipoden Schleiermacher sind als prägende Hintergrundserfahrungen der Bach- und Händelrezeption noch weiter zu erörtern.

Schließlich dürfen auch Fragen dessen, was man innerjüdischen Antisemitismus oder jüdischen Selbsthaß zu nennen pflegt, nicht ausgeklammert werden, soll Fanny Hensel als Person und Künstlerin in einem umfassenden Sinne verstanden werden. Gerade hier eröffnet die Theorie der Biographik, soweit sie das Problem der Generationenschichtung in den Blick faßt, für die Geschichte der Familie Mendelssohn - und damit auch für Fanny Hensel selbst - fruchtbare Fragen. Zeigt es sich doch, daß die Kinder auf der Generationsebene von Felix und Fanny Mendelssohn Bartholdy im Unterschied zur Elterngeneration deren rigorose Abkehr vom jüdischen Erbe teilweise wieder rückgängig machten bzw. ihre jüdische Herkunft in einem verständnisvoll-bewahrenden Lichte betrachteten. So schreibt Rebecka, daß der tiefe Haß ihres Vaters gegen das

nig] Fanny Mendelssohn: D'après les mémoires de son fils, Paris 1888, wäre noch abzuklären, wie der Kontakt zwischen der Autorin und Herausgeberin und Sebastian Hensel zustande kam, erklärt doch Sergy in ihrem Vorwort, es handle sich um eine von Sebastian Hensel autorisierte Fassung. 
Judentum ihr "oft unangenehm" gewesen sei, ${ }^{8}$ und Vergleichbares dürfte auch auf Fanny Hensel zutreffen. Die aktuelle Antisemitismusforschung bietet zur Klärung solcher Fragen reiche Rahmenbedingungen, die es insbesondere erlauben werden, die Figur Fanny Hensels mit jüdischen Akkulturationsgeschichten wie denjenigen von Heinrich Heine und Rahel Varnhagen zu vergleichen - Personen, die im Laufe ihres Lebens bei aller Distanz zum Judentum durchaus ein gebrochenes Verhältnis zur Taufe und zum Christentum entwickelt hatten. Schließlich ließen sich in diesem Kontext auch die Spannungen zwischen der Familie Mendelssohn Bartholdy und der Familie Giacomo Meyerbeers im Lichte der Tatsache, daß dieser sich dezidiert zum Judentum bekannte, noch spezifischer zu untersuchen.

Berlin, den 3. Oktober 1998

Monika Schwarz-Danuser

8 Sebastian Hensel, ein Lebensbild aus Deutschlands Lehrjahren, mit einem Vorwort hg. v. Paul Hensel, Berlin ${ }^{2} 1904$, S. 185. Im Brief, den Rebecka Dirichlet an ihren Neffen Sebastian am 4. April 1855 schrieb, heißt es: Ich habe während meines Krankseins allerlei Bücher durchgeblättert, u. A. ein Buch von Auerbach, Dichter und Kaufmann; es ist aber, obgleich manches hübsche darin ist, zu jüdisch für einen Juden. Unsereins kann sich nicht Veilchen und Täubchen reizend denken, ich habe das ganze Buch durch gemauschelt. Außerdem halte ich es für ein verkehrtes Ding, das Leben eines bestußten Schwachmatikus einen dicken Band durch $z u$ beschreiben, der nicht klüger (der Mann nämlich) dadurch wird, daß er mit Moses Mendelssohn und Lessing verkehrt. Doch hat's mich persönlich interessiert, weil ich an viele Erzählungen von Vater und Mutter dabei gedacht habe, namentlich Vaters tiefen Haß gegen das Judentum, der mir oft unangenehm war, wieder verschiedentlich darin erklärt und motiviert gefunden habe. 